\title{
Form follows need
}

\author{
The history of physics publishing in the past century shows how the changing needs of the research \\ community shaped the dissemination of knowledge through scientific journals.
}

The pressure to publish is by no means new. In 1900, after being scooped by Pierre and Marie Curie on a radioactivity paper, Ernest Rutherford wrote': "I have to publish my present work as rapidly as possible in order to keep in the race." Rapid publication had already become an important factor in establishing scientific priority, especially in as hot a topic as radioactivity. But at the beginning of the twentieth century, there were few journals publishing on a weekly basis. One of them was Nature, which happened to fit well with Rutherford's agenda, as it published quickly and was read by a European (mainly British) audience that Rutherford was targeting to secure a position in a UK institution.

Rutherford started using the Letters to the Editor section - a column traditionally dedicated to comments on someone else's work - as a fast way of communicating his own research and making priority claims. In 1902 Rutherford's contributions to the column were already looking more like scientific papers rather than commentary pieces (pictured). The practice proved quite successful and was soon adopted by Rutherford's collaborators and students, notably Otto Hahn, Niels Bohr and James Chadwick. By the 1930s this approach had been taken up by others, including Otto Frisch and Lise Meitner, and the Copenhagen school of physicists working with Bohr. Rutherford's pragmatic choice of a publishing venue spread and eventually shaped the Nature column as an important venue for communicating the latest research ${ }^{1,2}$. This was subsequently adopted by other disciplines and evolved into the Letter format surviving today in Nature and inherited by Nature Physics.

Perhaps inspired by the success of the format, John T. Tate, the editor of Physical Review, also introduced a section called Letters to the Editor in $1929^{3}$; the section subsequently became so successful that in 1958 it took off on its own in the form of Physical Review Letters. Having realized the challenge posed by the ever-growing number of publications and increasing specialization, Tate also looked into creating a new type of journal - one that published carefully synthesized reviews of emerging fields ${ }^{4}$. Although it initially received mixed responses, Tate's new publication, Reviews of Modern Physics, catered to the need for overviews of different fast-moving topics and eventually became the authoritative forum we know today.

The increasing internationalization of science raised the profile of English publications, forcing older journals such as Annalen der Physik to start publishing in both English and German. It also contributed to the exponential growth of publications requiring physicists to "cope with scientific literature by the ton"

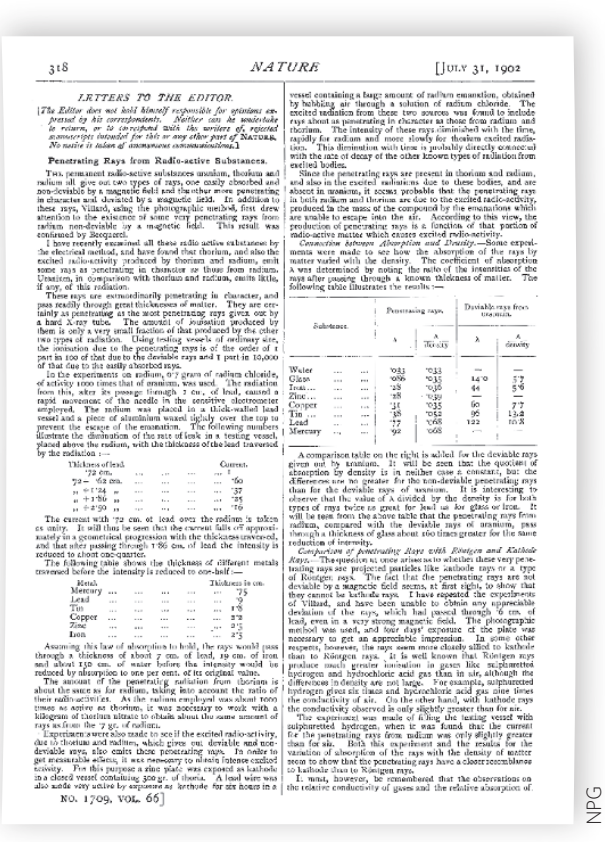

In the mid-1950s, Samuel Goudsmit, editor of Physical Review, was finding the issues of his journal "almost too bulky to carry" American physicists had already been debating ways of easing the problem of the increasing size of Physical Review. Ideas ranged from printing a weekly newspaper containing only the titles and abstracts of received papers, having a 'greatest hits' journal or a 'reader's digest', to dividing Physical Review into two separate sections for experimentalists and theorists ${ }^{5}$. More serious consideration was given to splitting Physical Review into sections covering different areas of physics, a development that eventually took place in steps: first in
1963 (two sections, one on solid-state and atomic physics and one on nuclear and high-energy physics) and then in 1966 when the two sections were divided again in half. By 1970 Physical Review was clearly divided into four independent biweekly journals. Other journals had to follow similar steps; Zeitschrift für Physik split into three journals around the same time.

Unlike Rutherford, who shipped his communications from his laboratory at McGill University in Canada across the Atlantic to the Nature office in London, in the 1990s physicists were able to share their results via e-mail and the World Wide Web. In 1991 Paul Ginsparg created the Los Alamos National Laboratory preprint archive, now known as the arXiv. The preprint server not only addressed the decades-old need for fast communication to peers, but also the new demand for open access. As an alternative, or rather a complement, to traditional peer-reviewed journals, the arXiv became immensely popular: it now consists of well over a million papers, and 8,000-9,000 new manuscripts are posted monthly.

We tend to think that high-visibility journals shape the landscape of research or individual researchers' careers, but historical evidence shows the opposite is also true. A scientist's agenda can shape a journal and the practical needs of the research community lead to new formats of the scientific journals or the creation of new outlets. The beginning of the twenty-first century is marked by the use of increasingly large datasets and a strong drive for open science. We have already witnessed the rise of open access mega-journals (such as PLoS ONE and Scientific Reports) and arXiv overlay journals (such as Discrete Analysis, recently launched by Timothy Gowers, a Cambridge-based mathematician). Their impact on scientific publishing, and on physics in general, remains to be seen, but no doubt the requirements of practicing scientists will lead the way.

\footnotetext{
References

1. Baldwin, M. Brit. J. Hist. Sci. 47, 257-279 (2014).

2. Baldwin, M. Making Nature (Univ. Chicago Press, 2015).

3. Lalli, R. Notes Rec. http://doi.org/bddf (2016).

4. Lalli, R. Ann. Phys. 526, A83-A87 (2014).

5. Kaiser, D. Osiris 27, 276-302 (2012).
} 